MATHEMATICS OF COMPUTATION

Volume 67, Number 222, April 1998, Pages 479-499

S 0025-5718(98)00946-6

\title{
A SPACE-TIME FINITE ELEMENT METHOD FOR THE NONLINEAR SCHRÖDINGER EQUATION: THE DISCONTINUOUS GALERKIN METHOD
}

\author{
OHANNES KARAKASHIAN AND CHARALAMBOS MAKRIDAKIS
}

\begin{abstract}
The convergence of the discontinuous Galerkin method for the nonlinear (cubic) Schrödinger equation is analyzed in this paper. We show the existence of the resulting approximations and prove optimal order error estimates in $L^{\infty}\left(L^{2}\right)$. These estimates are valid under weak restrictions on the space-time mesh.
\end{abstract}

\section{INTRODUCTION}

We consider the nonlinear Schrödinger equation (NLS)

$$
\begin{aligned}
& u_{t}=\mathrm{i} \Delta u+\mathrm{i} \lambda|u|^{2} u, \quad \text { in } \Omega \times[0, T], \\
& u=0, \quad \text { on } \partial \Omega \times[0, T], \\
& u(\cdot, 0)=u^{0} \quad \text { in } \Omega,
\end{aligned}
$$

where $\Omega$ is a bounded domain in $\mathbb{R}^{2}, u$ is a complex-valued function defined on $\bar{\Omega} \times[0, T]$ and $\lambda$ is a real parameter. We study the convergence of a space-time finite element method for (1.1), namely the discontinuous Galerkin method. The NLS equation arises, posed often as an initial value problem, in various areas of Mathematical Physics. For example, in one space dimension, it arises as an envelope equation in water wave theory $[\mathrm{N}],[\mathrm{W}]$. In two space dimensions, it occurs in nonlinear optics where it describes the propagation of electromagnetic beams in media whose index of refraction depends on the amplitude of the field in a simple nonlinear way [CGT], [T]. Since many interesting solutions are exponentially decaying at infinity, it is a standard practice to couch numerical schemes in the setting of the boundary value formulation (1.1).

Recently, increasing attention has been given to finite element methods in both space and time variables for evolution problems. One reason for considering such methods is the need for flexible schemes suitable for computations on unstructured meshes. These schemes are of particular interest for effective numerical computations for (1.1), since this equation admits solutions that form singularities in finite time, cf., e.g., [RR], [S]. Therefore the choice of appropriate adaptive methods seems to be a natural one in this case. As a first step, in this work we prove a priori error estimates that guarantee the convergence of a class of discontinuous Galerkin methods to the solution of (1.1) under weak restrictions on the variation of the (space-time) mesh. We use a rather general approach which can be applied

Received by the editor February 19, 1996 and, in revised form, October 25, 1996.

1991 Mathematics Subject Classification. Primary 65M60, 65M12.

(C)1998 American Mathematical Society 
to analyze the convergence behaviour of the discontinuous Galerkin method for hyperbolic type problems.

The discontinuous Galerkin method has been studied extensively in recent years for parabolic problems in Eriksson, Johnson and Thomée [EJT], Eriksson and Johnson [EJ1], [EJ2], [EJ3]. The method in the particular form that we consider in this paper was previously considered for parabolic problems in [EJ1], [EJ2] and belongs to the class of methods introduced in $[\mathrm{J}]$ for a parabolic problem in a variable domain; the discontinuous Galerkin method for time-dependent problems was proposed in $[\mathrm{LR}]$.

One of the reasons for considering the discontinuous Galerkin method in this paper is our intention to investigate the application of this method to a model problem which requires computations on unstructured meshes but on the other hand, does not have "strong" stability properties and hence the theory of Eriksson and Johnson is not applicable. In this light our results are compared with the corresponding results of [EJ1], [EJ2], see below. Note also that a priori error estimates for linear hyperbolic problems are derived in Bales and Lasiecka [BL], French and Peterson [FP], considering the continuous Galerkin method. Our intention is to apply both methods to (1.1), since we have no reason, a priori, to prefer one over the other, and also to see how these methods compare in terms of the flexibility of the selection of the space-time mesh, and the convergence results. In a forthcoming work, we shall report on the convergence analysis for the continuous Galerkin method and present numerical experiments comparing the performance of the two methods. In a recent paper, Dörfler [Dr] has derived a posteriori error estimates and proposed an adaptive algorithm based on the continuous Galerkin method for a linear Schrödinger equation.

We now introduce the discontinuous Galerkin method for the problem (1.1): Let $0=t^{0}<t^{1}<\cdots<t^{N}=T$ be a partition of $[0, T]$, and

$$
I_{n}=\left(t^{n}, t^{n+1}\right], \quad k_{n}=t^{n+1}-t^{n} .
$$

We associate a partition $\mathcal{T}_{h n}$ of $\Omega$ and a finite element space $S_{h}^{n}$ to each interval $I_{n}:$

$$
S_{h}^{n}=\left\{\chi \in H_{0}^{1}(\Omega):\left.\chi\right|_{K} \in \mathbb{P}_{r-1}(K), K \in \mathcal{T}_{h n}\right\},
$$

where $\mathbb{P}_{p}(S)$ is the space of polynomials of degree $p$. We also associate a space $S_{h}^{-1}$ with $\left\{t^{0}\right\}$; for simplicity we take $S_{h}^{-1}=S_{h}^{0}$. In the sequel we shall denote by $K$ the elements of the partition $\mathcal{T}_{h n}$. Also $h_{K}$ stands for the diameter of the element $K$, and $h_{n}=\max _{K \in \mathcal{T}_{h n}} h_{K}$.

Following standard notation, we let $H^{\ell}(S)$ be the (complex) Sobolev space of order $\ell$, and we denote its norm by $\|\cdot\|_{\ell, S}\left(\|\cdot\|_{\ell}\right.$ if $\left.S=\Omega\right)$. Also $(\cdot, \cdot)$ denotes the inner product, and $\|\cdot\|$ the corresponding norm on $L^{2}(\Omega) ;\|\cdot\|_{\infty}$ denotes the norm of $L^{\infty}(\Omega)$ and $\|\cdot\|_{1, \infty}$ the norm of $W^{1, \infty}(\Omega)$.

Now let $V_{h k}=V_{h k}(q), q$ positive integer, be the space of piecewise polynomial functions $\varphi: \Omega \times(0, T] \rightarrow \mathbb{C}$ of the form: $\left.\varphi\right|_{\Omega \times I_{n}}=\sum_{j=0}^{q-1} t^{j} \chi_{j}(x), \quad \chi_{j} \in S_{h}^{n}$. Hence, the functions of $V_{h k}$ are, for each $t \in I_{n}$, elements of $S_{h}^{n}$ and for each $x \in \Omega$ piecewise polynomial functions of degree $q-1$ with possible discontinuities at the nodes $t^{n}, n=0, \ldots, N-1$. Let also $V_{h k}^{n}=\left\{\left.\varphi\right|_{\Omega \times I_{n}}: \varphi \in V_{h k}\right\}$.

We assume that (1.1) admits a unique smooth solution on $[0, T]$. We define the approximations $U \in V_{h k}$ to the solution $u$ of (1.1) as follows: Find $U \in V_{h k}$ 
satisfying

$$
\begin{aligned}
\left(U^{n+1}, \phi^{n+1}\right)-\int_{I_{n}}\left(U, \phi_{t}\right)+\mathrm{i} \int_{I_{n}}(\nabla U, \nabla \phi) d t-\mathrm{i} \lambda \int_{I_{n}}\left(|U|^{2} U, \phi\right) d t \\
=\left(U^{n}, \phi^{n+}\right), \quad \forall \phi \in V_{h k}^{n}, \quad n=0, \ldots, N-1,
\end{aligned}
$$

where $U^{n}=U\left(t^{n}\right), v^{n+}:=\lim _{t \rightarrow t^{n}+} v(t)$ and we have set $U^{0}=u^{0}$.

In the sequel we shall make frequent use of the following notation: We denote by $\|\cdot\|_{n}$ and by $\max _{I_{n}}\|\cdot\|$ the norms of $L^{2}\left(I_{n}, L^{2}(\Omega)\right)$ and $C\left(\bar{I}_{n}, L^{2}(\Omega)\right)$ respectively, i.e.,

$$
\|v\|_{n}:=\left(\int_{I_{n}}\|v(t)\|^{2} d t\right)^{1 / 2} .
$$

Also for $s, m=0,1, \ldots$, and $v \in H^{m}(\Omega)$ we let

$$
\left\|h_{n}^{s} v\right\|_{m, h}:=\left(\sum_{K \in \mathcal{T}_{h n}} h_{K}^{2 s}\|v\|_{m, K}^{2}\right)^{1 / 2} .
$$

Summary of results. As an intermediate step towards proving the convergence of (1.2) for (1.1) we introduce the modified scheme:

$$
\begin{aligned}
\left(U^{n+1}, \phi^{n+1}\right)-\int_{I_{n}}\left(U, \phi_{t}\right)+\mathrm{i} \int_{I_{n}}(\nabla U, \nabla \phi) d t-\mathrm{i} \lambda \int_{I_{n}}(f(U), \phi) d t \\
=\left(U^{n}, \phi^{n+}\right), \quad \forall \phi \in V_{h k}^{n}, \quad n=0, \ldots, N-1,
\end{aligned}
$$

where $U^{0}=u^{0}$ and $f=f(z), f: \mathbb{C} \rightarrow \mathbb{C}$, is an appropriate globally Lipschitz continuous function (cf. Section 4), such that $f(z)=|z|^{2} z$ in a ball with a radius that depends on the solution $u$ of (1.1). In particular, $u$ is in that ball. We first derive an estimate of the error of the approximations defined by (1.3) and then, under some assumptions on the mesh, we show that this satisfies (1.2) as well and therefore the error estimate remains valid for the solution of (1.2). Sections 2 and 3 are devoted to the analysis of (1.3): In Section 2 we establish the existence of a solution of (1.3). The existence as well as convergence proofs rest on properties of the Lagrange interpolating polynomials associated with the Radau interpolation.

In Section 3 we present the basic error analysis in $L^{\infty}\left(L^{2}(\Omega)\right)$. The main result of this section can be stated roughly as follows, cf. Theorem 3.1: If $u$ is the solution of (1.1) and $U \in V_{h k}$ is the solution of (1.3), then

$$
\begin{gathered}
\max _{t \in[0, T]}\|u(t)-U(t)\| \leq C \max _{m} k_{m}^{q} \max _{I_{m}}\left(\left\|u^{(q)}\right\|+\left\|u^{(q+1)}\right\|+\left\|\Delta u^{(q)}\right\|\right) \\
+C \max _{m} \max _{I_{m}}\left(\left\|h_{m}^{r} u_{t}\right\|_{r, h}+\left\|h_{m}^{r} u\right\|_{r, h}\right) \\
+C \mathcal{N}_{C} \max _{m}\left\|J\left[\omega^{m}\right]\right\|
\end{gathered}
$$

where $\mathcal{N}_{C}$ denotes the number of times where $S_{h}^{j} \neq S_{h}^{j-1}, j=1, \ldots N-1$, and $J\left[\omega^{n}\right]=\omega^{n+}-\omega^{n}$ is the jump $\left(P_{E}^{n}-P_{E}^{n-1}\right) u\left(t_{n}\right)$ of the elliptic projection $\omega(t)=$ $P_{E}^{n} u(t)$ at $t_{n}$. Note that the constant in this estimate is of the form $C=c e^{c T}$. We have also assumed that the error of the elliptic projection in the $L^{2}$-norm can be estimated by the local-type bound $\left\|h_{m}^{r} u\right\|_{r, h}$, cf. (3.4b) and Remark 3.1. The same estimate, (1.4), is also valid for the corresponding linear problem, $f(u)=$ $g(x, t)$. The relation (1.4) is an optimal-order error estimate provided we do not change the spaces $S_{h}^{n}$ too often - compare with the results of Dupont [D]; but even in this case (1.4) guarantees convergence, since $\left\|J\left[\omega^{m}\right]\right\|$ is always bounded by 
$\left\|h_{m-1}^{r} u\right\|_{r, h}+\left\|h_{m}^{r} u\right\|_{r, h}$. (We have some preliminary results showing that $\left\|J\left[\omega^{m}\right]\right\|$ can be estimated more accurately in some important special cases). Note, however that (1.4) is not optimal, compared with standard elliptic finite element estimates, in terms of the regularity required for the exact solution. The results of Eriksson and Johnson, [EJ1], [EJ2], show that optimal order-regularity estimates (up to a logarithmic factor) are valid for the corresponding discontinuous Galerkin method for linear parabolic problems, provided in each time slab either $S_{h}^{n} \subset S_{h}^{n-1}$ or $k_{n} \geq c h_{n}^{2}$. The approach in [EJ1], [EJ2] is based on establishing "strong" stability estimates for an appropriate discrete dual problem. These estimates depend in an essential manner on the parabolic character of the problem considered in [EJ1], [EJ2]. Our approach is different and is based on a combination of finite element and finite difference techniques, taking full advantage of appropriate energy-type estimates and of useful properties of Lagrange interpolating polynomials at the Radau points of each $I_{n}$. Note that in our case the estimate (1.4) is valid without any restrictive assumptions on the choice of $S_{h}^{n}, n=0, \ldots, N-1$, cf. also Remark 3.1. We refer also to $[\mathrm{HH}],[\mathrm{Jh}]$ where a discontinuous Galerkin method is analyzed for linear second-order hyperbolic problems.

In Section 4, we prove that if $U$ is the solution of (1.3), then $\max _{[0, T]}\|\nabla U(t)\|$ can be controlled under some assumptions on the choice of the space-time mesh. In view of an inverse inequality, and provided that for each $n, 1 \leq n \leq N-1$, there holds

$$
\begin{aligned}
& S_{h}^{n-1} \subset S_{h}^{n} \\
& \left\|\nabla P^{n} v\right\| \leq C_{P}\|\nabla v\|, \quad \forall v \in H_{0}^{1}(\Omega) \quad \text { or } \quad k_{n} \geq C_{N}\left(k^{2 q}+h^{2 r}\right),
\end{aligned}
$$

we prove that the solution $U$ of (1.3) is a solution of (1.2) as well (Theorem 4.1) and thus satisfies the error bound (1.4). Here

$$
k=\max _{0 \leq n \leq N-1} k_{n}, h=\max _{\substack{K \in \mathcal{T}_{h} \\ 0 \leq n \leq N-1}} h_{K}, \underline{h}=\min _{\substack{K \in \mathcal{T}_{h n} \\ 0 \leq n \leq N-1}} h_{K}, C_{N}=c|\ln (\underline{h})|,
$$

and $P^{n}$ denotes the $L^{2}$-projection into $S_{h}^{n}$, cf. Remark 4.1. Note that our results hold if $\Omega \subset \mathbb{R}$ and can be extended if $\Omega \subset \mathbb{R}^{3}$, by assuming (in Section 4 ) stronger meshconditions. For convergence of finite element schemes to (1.1) we refer to $[\mathrm{ADK}]$ and the references therein.

Finally, let us note that the following $\sigma$-order superconvergence result at the time nodes holds, [KM1]: Under the assumptions of section 3 and section 4 and assuming that $u$ has sufficient regularity

$$
\begin{aligned}
\left\|u\left(t^{n+1}\right)-U\left(t^{n+1}\right)\right\| \leq & C \max _{m \leq n} C_{1}(m, u) k_{m}^{\sigma}+C \max _{m \leq n} C_{2}(m, u) h_{m}^{r} \\
& +C \mathcal{N}_{C} \max _{m \leq n}\left\|J\left[\omega^{m}\right]\right\|,
\end{aligned}
$$

where $\sigma=2 q-1$ if $\Omega$ is polyhedral and $\sigma=\min \{q+2,2 q-1\}$, otherwise; $C_{1}(m, u)$ and $C_{2}(m, u)$ are constants that depend on $m$ and $u$ but are independent of $h$ and $k$.

We next present some concluding remarks concerning interesting connections between the discontinuous Galerkin method and the class of Radau IIA implicit Runge-Kutta (IRK) methods, cf., e.g., [DV], [HW]. The replacement of the time integrals in (1.2) by a $q$-point Radau quadrature formula (which turns out to be exact except for the nonlinear term) transforms (1.2) into a $q$-stage Radau IIA method. The application of this as well as other classes of IRK methods to (1.1) can 
be considered as a finite-difference approach to time-stepping and was analyzed in [KAD] in the case where $S_{h}^{n}=S_{h}, n=0, \ldots, N-1$. This close relationship is made evident here through the use of the Lagrange interpolating polynomials associated with the Radau abscissas. Indeed, the proof of Lemma 2.1 below contains arguments similar to those used to prove the BSI stability of the Radau IIA methods, cf. [DV, pp. 162-164]. On the other hand, while the convergence of IRK methods relies on the concept of Algebraic Stability as well as a set of consistency conditions known as the Simplifying Assumptions, the convergence analysis presented herein is free from such considerations and flows naturally from the basic properties of the method itself. This is perhaps an indication that the discontinuous Galerkin method and by extension other space-time Galerkin methods capture the essence of time-stepping at a deeper level.

Let us note finally that an approach similar to the present paper can be taken to analyze the convergence of the discontinuous Galerkin method for general semilinear second-order hyperbolic problems.

\section{Existence}

For each $q \geq 1$, we consider the integration rule,

$$
\int_{0}^{1} g(\tau) d \tau \cong \sum_{j=1}^{q} w_{j} g\left(\tau_{j}\right), \quad 0<\tau_{1}<\cdots<\tau_{q}=1,
$$

which is exact for all polynomials of degree $\leq 2 q-2$. These quadrature rules are known as Radau methods, cf. [DR], and play a fundamental role in the analysis presented herein.

For fixed $q \geq 1$, let $\left\{\ell_{i}\right\}_{i=1}^{q}$ be the Lagrange polynomials associated with the abscissae $\tau_{1}, \ldots, \tau_{q}$, i.e.

$$
\ell_{i}(\tau)=\prod_{\substack{j=1 \\ i \neq j}}^{q} \frac{\left(\tau-\tau_{j}\right)}{\left(\tau_{i}-\tau_{j}\right)}
$$

Using the linear transformation $t=t^{n}+\tau k_{n}$ that maps [0,1] onto $\bar{I}_{n}$, we adapt the quadrature rule (2.1) to the interval $\bar{I}_{n}$ by defining its abscissae and weights as follows:

$$
\begin{aligned}
& t^{n, j}=t^{n}+\tau_{j} k_{n}, \quad j=1, \ldots, q \quad\left(t^{n, q}=t^{n+1}\right), \\
& \ell_{n, i}(t)=\ell_{i}(\tau), \quad t=t^{n}+\tau k_{n} \\
& w_{n, i}=\int_{t^{n}}^{t^{n+1}} \ell_{n, i}(t) d t=k_{n} \int_{0}^{1} \ell_{i}(\tau) d \tau=k_{n} w_{i}, \quad i=1, \ldots, q .
\end{aligned}
$$

Then $\left.U\right|_{I_{n}}$ is uniquely determined by the functions $U^{n, j}=U^{n, j}(x) \in S_{h}^{n}$, such that

$$
U(x, t)=\sum_{j=1}^{q} \ell_{n, j}(t) U^{n, j}(x), \quad(x, t) \in \Omega \times I_{n} .
$$


Now, if $\psi=\psi(x), \psi \in S_{h}^{n}$, the function $\varphi=\ell_{n, i} \psi$ is an element of $V_{h k}^{n}$ and (1.3) is equivalent to

$$
\begin{aligned}
& \delta_{q i}\left(U^{n, q}, \psi\right)-\sum_{j=1}^{q} w_{n, j} \ell_{n, i}^{\prime}\left(t^{n, j}\right)\left(U^{n, j}, \psi\right)+\mathrm{i} k_{n} w_{i}\left(\nabla U^{n, i}, \nabla \psi\right) \\
& (2.5) \quad-\mathrm{i} \lambda \int_{I_{n}} \ell_{n, i}(t)(f(U), \psi) d t=\ell_{n, i}\left(t^{n}\right)\left(U^{n}, \psi\right), \quad i=1, \ldots, q, \quad \psi \in S_{h}^{n} .
\end{aligned}
$$

We consider the $q \times q$ matrices $\mathcal{N}, \mathcal{M}$ defined by

$$
\begin{aligned}
& \mathcal{N}_{i j}=w_{n, j} \ell_{n, i}^{\prime}\left(t^{n, j}\right)=w_{j} \ell_{i}^{\prime}\left(\tau_{j}\right), \\
& \mathcal{M}=e_{q} e_{q}^{T}-\mathcal{N}, \quad e_{q}^{T}=(0, \ldots, 1) \in \mathbb{R}^{q} .
\end{aligned}
$$

It is clear that $\mathcal{N}, \mathcal{M}$ are independent of $k_{n}$ and if $Y=\left(y^{n, 1}, \ldots, y^{n, q}\right) \in \mathbb{R}^{q}$, then

$$
Y^{T} \mathcal{M} Y=\sum_{i=1}^{q} \delta_{q i} y^{n, q} y^{n, i}-\sum_{i, j=1}^{q} w_{n, j} \ell_{n, i}^{\prime}\left(t^{n, j}\right) y^{n, j} y^{n, i} .
$$

A crucial result for the proof of existence, as well as for the rest of the paper, is that there exists a positive, diagonal matrix $D$ such that $D^{-1 / 2} \mathcal{M} D^{1 / 2}$ is positive definite. Before proving this, we note that since $w_{j}=\int_{0}^{1} \ell_{j}^{2}(t) d t$, the weights $w_{j}$ are positive.

Lemma 2.1. Let $\widetilde{\mathcal{M}}$ be the matrix

$$
\widetilde{\mathcal{M}}=D^{-1 / 2} \mathcal{M} D^{1 / 2}, \quad D=\operatorname{diag}\left\{\tau_{1}, \tau_{2}, \ldots, \tau_{q}\right\} .
$$

Then with $\alpha:=\frac{1}{2} \min \left\{\frac{w_{1}}{\tau_{1}}, \ldots, \frac{w_{q-1}}{\tau_{q-1}}, 1+w_{q}\right\}>0$, there holds

$$
\mathbf{x}^{T} \widetilde{\mathcal{M}} \mathbf{x} \geq \alpha|\mathbf{x}|^{2}=\alpha\left(\sum_{i=1}^{q} x_{i}^{2}\right), \quad \forall \mathbf{x} \in \mathbb{R}^{q} .
$$

Proof. We will first prove that

$$
\mathcal{N} D+D \mathcal{N}^{T}=\operatorname{diag}\left\{-w_{1}, \ldots,-w_{q-1}, 1-w_{q}\right\} .
$$

For this let

$$
\gamma_{i j}(\tau)=\tau \ell_{i}^{\prime}(\tau) \ell_{j}(\tau) .
$$

Since the quadrature rule $(2.1)$ is exact on $\mathbb{P}_{2 q-2}$,

$$
\begin{aligned}
\int_{0}^{1} \gamma_{i j}(\tau) d \tau & =\sum_{m=1}^{q} w_{m} \tau_{m} \ell_{i}^{\prime}\left(\tau_{m}\right) \ell_{j}\left(\tau_{m}\right) \\
& =w_{j} \tau_{j} \ell_{i}^{\prime}\left(\tau_{j}\right)=(\mathcal{N} D)_{i j} .
\end{aligned}
$$

On the other hand, integrating by parts

$$
\int_{0}^{1} \gamma_{i j}(\tau) d \tau=\ell_{i}(1) \ell_{j}(1)-\int_{0}^{1} \ell_{i}(\tau) \ell_{j}(\tau) d \tau-\int_{0}^{1} \gamma_{j i}(\tau) d \tau,
$$

i.e.,

$$
\begin{aligned}
(\mathcal{N} D)_{i j}+(\mathcal{N} D)_{j i} & =\ell_{i}(1) \ell_{j}(1)-\int_{0}^{1} \ell_{i}(\tau) \ell_{j}(\tau) d \tau \\
& =\ell_{i}(1) \ell_{j}(1)-\delta_{i j} w_{j}, \quad i, j=1, \ldots, q,
\end{aligned}
$$


and (2.9) follows. Now let $\mathbf{x} \in \mathbb{R}^{q}$. Relation (2.9) and the fact that $\tau_{q}=1$ imply,

$$
\begin{aligned}
\mathbf{x}^{T} \widetilde{\mathcal{M}} \mathbf{x} & =x_{q}^{2}-\mathbf{x}^{T} D^{-1 / 2} \mathcal{N} D^{1 / 2} \mathbf{x} \\
& =x_{q}^{2}-\frac{1}{2} \mathbf{x}^{T}\left[D^{-1 / 2} \mathcal{N} D^{1 / 2}+D^{1 / 2} \mathcal{N}^{T} D^{-1 / 2}\right] \mathbf{x} \\
& =x_{q}^{2}-\frac{1}{2} \mathbf{x}^{T} D^{-1 / 2}\left[\mathcal{N} D+D \mathcal{N}^{T}\right] D^{-1 / 2} \mathbf{x} \\
& =x_{q}^{2}-\frac{1}{2} \mathbf{x}^{T} \operatorname{diag}\left\{-\frac{w_{1}}{\tau_{1}}, \ldots,-\frac{w_{q-1}}{\tau_{q-1}}, \frac{1-w_{q}}{\tau_{q}}\right\} \mathbf{x} \\
& \geq \alpha|\mathbf{x}|^{2} .
\end{aligned}
$$

We will prove existence by exhibiting $\left\{\widetilde{U}^{n, j}\right\}_{j=1}^{q}$, where $\widetilde{U}^{n, j}=\tau_{j}^{-1 / 2} U^{n, j} \in S_{h}^{n}$, i.e., in view of $(2.4)$,

$$
U(x, t)=\sum_{j=1}^{q} \tau_{j}^{1 / 2} \ell_{n, j}(t) \widetilde{U}^{n, j}(x), \quad(x, t) \in \Omega \times I_{n} .
$$

To take advantage of the positivity of $\widetilde{\mathcal{M}}$ we choose $\varphi=\tau_{i}^{-1 / 2} \ell_{n, i} \psi$ in (1.3), where $\psi \in S_{h}^{n}$, and we use the expression (2.10) for $U$ to obtain, cf. (2.5),

$$
\begin{aligned}
\delta_{q i}\left(\widetilde{U}^{n, q}, \psi\right) & -\sum_{j=1}^{q} w_{n, j} \ell_{n, i}^{\prime}\left(t^{n, j}\right) \tau_{j}^{1 / 2} \tau_{i}^{-1 / 2}\left(\widetilde{U}^{n, j}, \psi\right) \\
& +\mathrm{i} k_{n} w_{i}\left(\nabla \widetilde{U}^{n, i}, \nabla \psi\right)-\mathrm{i} \lambda \int_{I_{n}} \tau_{i}^{-1 / 2} \ell_{n, i}(f(U), \psi) d t \\
& -\tau_{i}^{-1 / 2} \ell_{n, i}\left(t^{n}\right)\left(U^{n}, \psi\right)=0, \quad i=1, \ldots, q, \psi \in S_{h}^{n} .
\end{aligned}
$$

We are ready to prove the following

Theorem 2.1. Let $U^{n}$ be given in $S_{h}^{n-1}$, then for $k_{n}$ sufficiently small there exists $\left\{\widetilde{U}^{n, j}\right\}_{j=1}^{q}$ in $\left(S_{h}^{n}\right)^{q}$ satisfying (2.11). Therefore equation (1.3) has a solution $U \in$ $V_{h k}^{n}$. Furthermore $U$ is unique.

Proof. The vector space $\left(S_{h}^{n}\right)^{q}$ is a finite dimensional Hilbert space equipped with the inner product

$$
((X, \Psi))=\sum_{i=1}^{q}\left(\chi_{i}, \psi_{i}\right), \quad X=\left(\chi_{1}, \ldots, \chi_{q}\right)^{T}, \quad \Psi=\left(\psi_{1}, \ldots, \psi_{q}\right)^{T} \in\left(S_{h}^{n}\right)^{q} .
$$

We denote the associated norm $\left(\sum_{i=1}^{q}\left\|\chi_{i}\right\|^{2}\right)^{\frac{1}{2}}$ by $\|\cdot\|$. We shall use a variation of Brouwer's fixed point theorem, cf. [Br], to show that the map $F:\left(S_{h}^{n}\right)^{q} \rightarrow\left(S_{h}^{n}\right)^{q}$ defined by

$$
\begin{aligned}
\left(F(V)_{i}, \psi\right) & =\delta_{q i}\left(v_{q}, \psi\right)-\sum_{j=1}^{q} w_{j} \ell_{i}^{\prime}\left(\tau_{j}\right) \tau_{j}^{1 / 2} \tau_{i}^{-1 / 2}\left(v_{j}, \psi\right) \\
& +\mathrm{i} k_{n} w_{i}\left(\nabla v_{i}, \nabla \psi\right)-\mathrm{i} \lambda \int_{I_{n}} \tau_{i}^{-1 / 2} \ell_{n, i}\left(f\left(\sum_{j=1}^{q} \tau_{j}^{1 / 2} \ell_{n, j} v_{j}\right), \psi\right) d t \\
& -\tau_{i}^{-1 / 2} \ell_{n, i}\left(t^{n}\right)\left(U^{n}, \psi\right), \quad \forall \psi \in S_{h}^{n}, \quad i=1, \ldots, q
\end{aligned}
$$


has a root in $\left(S_{h}^{n}\right)^{q}$, i.e. that $(2.11)$ has a solution. Note that since $f: \mathbb{C} \rightarrow \mathbb{C}$ is continuous, $F$ is continuous and therefore, cf. [Br], it suffices to show the existence of a constant $\beta>0$ such that

$$
\operatorname{Re}((F(V), V)) \geq 0, \quad \text { for all } V \in\left(S_{h}^{n}\right)^{q} \text { such that }\|V\|=\beta \text {. }
$$

To this end, set $\psi=v_{i}$ in (2.12) and sum from $i=1$ to $q$, to obtain:

$$
\begin{aligned}
\operatorname{Re}((F(V), V)) & \geq \operatorname{Re}\left\{\sum_{i=1}^{q} \delta_{q i}\left(v_{q}, v_{i}\right)-\sum_{i, j=1}^{q} w_{j} \ell_{i}^{\prime}\left(\tau_{j}\right) \tau_{j}^{1 / 2} \tau_{i}^{-1 / 2}\left(v_{j}, v_{i}\right)\right\} \\
& -|\lambda| \int_{I_{n}} \sum_{i=1}^{q}\left|\tau_{i}^{-1 / 2} \ell_{n, i}\left(f\left(\sum_{j=1}^{q} \tau_{j}^{1 / 2} \ell_{n, j} v_{j}\right), v_{i}\right)\right| d t \\
& -\sum_{i=1}^{q}\left|\tau_{i}^{-1 / 2} \ell_{n, i}\left(t^{n}\right)\left(U^{n}, v_{i}\right)\right| .
\end{aligned}
$$

Now, it is straightforward to see that Lemma 2.1 implies

$$
\begin{aligned}
\operatorname{Re}\left\{\sum_{i=1}^{q} \delta_{q i}\left(v_{q}, v_{i}\right)\right. & \left.-\sum_{i, j=1}^{q} w_{j} \ell_{i}^{\prime}\left(\tau_{j}\right) \tau_{j}^{1 / 2} \tau_{i}^{-1 / 2}\left(v_{j}, v_{i}\right)\right\} \\
& =\operatorname{Re}((\widetilde{M} V, V)) \geq \alpha\|V\|^{2},
\end{aligned}
$$

where $\alpha>0$ is the constant of (2.8). On the other hand, from (4.2) it follows that

$$
|\lambda| \int_{I_{n}} \sum_{i=1}^{q}\left|\tau_{i}^{-\frac{1}{2}} \ell_{n, i}\left(f\left(\sum_{j=1}^{q} \tau_{j}^{1 / 2} \ell_{n, j} v_{j}\right), v_{i}\right)\right| d t \leq \widetilde{c}_{1} k_{n}\|V\|^{2}, \quad \widetilde{c}_{1}=c c_{1} .
$$

Also

$$
\sum_{i=1}^{q}\left|\tau_{i}^{-1 / 2} \ell_{n, i}\left(t^{n}\right)\left(U^{n}, v_{i}\right)\right| \leq c_{2}\left\|U^{n}\right\|\|V\| .
$$

Combining (2.14-17) and assuming that $k_{n} \leq \frac{\alpha}{2 \widetilde{c}_{1}}$ we obtain

$$
\begin{aligned}
\operatorname{Re}((F(V), V)) & \geq\|V\|\left\{\left(\alpha-\widetilde{c}_{1} k_{n}\right)\|V\|-c_{2}\left\|U^{n}\right\|\right\} \\
& \geq\|V\|\left\{\frac{\alpha}{2}\|V\|-c_{2}\left\|U^{n}\right\|\right\},
\end{aligned}
$$

and therefore (2.13) holds for all $V$ such that

$$
\|V\|=\beta \geq \frac{2 c_{2}}{\alpha}\left\|U^{n}\right\|
$$

The proof of existence is now complete. Uniqueness is proved by similar arguments in view of (4.3).

\section{ERror ANALYSis in $L^{\infty}\left(L^{2}(\Omega)\right)$}

As usual we split the error $U-u=(U-W)+(W-u)$, where $W \in V_{h k}$ is an appropriately chosen function, and we estimate $E=U-W$ and $\rho=u-W$. We next define $W$ and derive the basic error equation for $E$ :

By $P_{E}^{n}$ we denote the elliptic projection operator $P_{E}^{n}: H_{0}^{1}(\Omega) \rightarrow S_{h}^{n}$ defined by

$$
\left(\nabla P_{E}^{n} v, \nabla \chi\right)=(\nabla v, \nabla \chi), \quad \forall \chi \in S_{h}^{n} .
$$


$\mathcal{I}_{q-1}^{n}$ is the usual Lagrange interpolation operator at the Radau points of $I_{n}=$ $\left(t_{n}, t_{n+1}\right]$, i.e. $\mathcal{I}_{q-1}^{n}: C\left(I_{n}\right) \rightarrow \mathbb{P}_{q-1}\left(I_{n}\right)$ is such that

$$
\left(\mathcal{I}_{q-1}^{n} y\right)\left(t^{n, j}\right)=y\left(t^{n, j}\right), \quad j=1, \ldots, q,
$$

where $t^{n, j}, j=1, \ldots, q$ are defined in $(2.3)$. Note that $\mathcal{I}_{q-1} u(x, \cdot) \in \mathbb{P}_{q-1}\left(I_{n}\right)$ and $\mathcal{I}_{q-1} u\left(x, t^{n+1}\right)=u\left(x, t^{n+1}\right), x \in \Omega$. It is clear that the function $W:(0, T] \rightarrow H_{0}^{1}(\Omega)$ defined by

$$
W(x, t)=\mathcal{I}_{q-1}^{n} P_{E}^{n} u(x, t), \quad(x, t) \in \Omega \times I_{n},
$$

is an element of $V_{h k}$. We denote its restriction to $I_{n},\left.W\right|_{I_{n}}$ again by $W$.

We assume that the family of spaces $S_{h}^{n}$ satisfy

$$
\left\|\nabla\left(v-P_{E}^{n} v\right)\right\| \leq c\left\|h_{n}^{s-1} v\right\|_{s, h}, \quad v \in H^{s} \cap H_{0}^{1}, 2 \leq s \leq r,
$$

and

$$
\left\|v-P_{E}^{n} v\right\| \leq c\left\|h_{n}^{s} v\right\|_{s, h}, \quad v \in H^{s} \cap H_{0}^{1}, \quad 2 \leq s \leq r,
$$

where $c$ is independent of $n$.

Remark 3.1. It is known that (3.4b) is valid in one dimension without any assumptions on the mesh, cf. [BO]. In higher dimensions (3.4b) is valid under appropriate local quasiuniformity conditions, cf. [EJ1], [EJ2] and their references and also [BS, Chapter 0] for a discussion of the one-dimensional case. Note that (3.4b) is not an essential assumption for our results to hold, in the sense that our estimates will remain valid if we replace the $\left\|h_{n}^{r} v\right\|_{r, h}$-like terms in Theorems 3.1 and 4.1 by terms that bound the error of the elliptic projection in the $L^{2}$-norm.

Standard approximation and stability results for the interpolation operator, [BS], [Ci] give,

$$
\|u-W\|_{n} \leq c k_{n}^{q}\left\|u^{(q)}\right\|_{n}+c k_{n}^{1 / 2} \max _{I_{n}}\left\|h_{n}^{s} u\right\|_{s, h}, \quad 2 \leq s \leq r,
$$

and

$$
\max _{I_{n}}\|u-W\| \leq c k_{n}^{q} \max _{I_{n}}\left\|u^{(q)}\right\|+c \max _{I_{n}}\left\|h_{n}^{s} u\right\|_{s, h}, \quad 2 \leq s \leq r .
$$

The basic error equation. The function $E=\left.E\right|_{I_{n}}$ satisfies,

$$
\begin{aligned}
& \left(E^{n+1}, \phi^{n+1}\right)-\int_{I_{n}}\left(E, \phi_{t}\right) d t+\mathrm{i} \int_{I_{n}}(\nabla E, \nabla \phi) d t-\mathrm{i} \lambda \int_{I_{n}}(f(U)-f(W), \phi) d t \\
& =\left(E^{n}, \phi^{n+}\right)-\left(W^{n+1}, \phi^{n+1}\right)+\int_{I_{n}}\left(W, \phi_{t}\right) d t+\left(W^{n}, \phi^{n+}\right) \\
& -\mathrm{i} \int_{I_{n}}(\nabla W, \nabla \phi)+\mathrm{i} \lambda \int_{I_{n}}(f(W), \phi) d t, \quad \forall \phi \in V_{h k}^{n}, n=0,1, \ldots, N-1,
\end{aligned}
$$

where we have set

$$
W^{0}=P_{E}^{0} u^{0}, \quad E^{0}=U^{0}-W^{0}=u^{0}-P_{E}^{0} u^{0} .
$$

We now introduce the functions $\omega, \eta$

$$
\begin{aligned}
\omega(x, t) & =P_{E}^{n} u(x, t), \\
\eta(x, t) & =(u-\omega)(x, t), \quad(x, t) \in \Omega \times I_{n}, \quad n=0, \ldots, N-1 .
\end{aligned}
$$

Obviously these functions are continuous with respect to $t$ in each time interval $I_{n}$, and have jump discontinuities at the points $t_{n}$, only if $S_{h}^{n-1} \neq S_{h}^{n}$. Recall that 
$\left.W\right|_{I_{n}}$ is the interpolant in $t$ of $\omega$ at the points $t^{n, j}$, and therefore using the notation introduced in the beginning of Section 2 we have

$$
\begin{aligned}
& W(x, t)=\sum_{j=1}^{q} \ell_{n, j}(t) \omega^{n, j}(x), \quad(x, t) \in \Omega \times I_{n}, \\
& \omega^{n, j}=\omega\left(\cdot, t^{n, j}\right)=P_{E}^{n} u^{n, j}=P_{E}^{n} u\left(\cdot, t^{n, j}\right), \quad j=1, \ldots, q .
\end{aligned}
$$

Also, cf. (2.4),

$$
\begin{aligned}
E(x, t) & =\sum_{j=1}^{q} \ell_{n, j}(t) E^{n, j}(x), \quad(x, t) \in \Omega \times I_{n}, \\
E^{n, j}(x) & =U^{n, j}-\omega^{n, j} .
\end{aligned}
$$

Using the above expressions in the equation (3.6) with test function $\phi=\ell_{n, i} \psi$, $\psi \in S_{h}^{n}$, we obtain the basic error equation for E, cf. (2.5),

$$
\begin{aligned}
& \delta_{q i}\left(E^{n, q}, \psi\right)-\sum_{j=1}^{q} w_{n, j} \ell_{n, i}^{\prime}\left(t^{n, j}\right)\left(E^{n, j}, \psi\right)+\mathrm{i} k_{n} w_{i}\left(\nabla E^{n, i}, \nabla \psi\right) \\
& \quad-\mathrm{i} \lambda \int_{I_{n}} \ell_{n, i}(f(U)-f(W), \psi) d t \\
& =\left(E^{n}, \ell_{n, i}\left(t^{n}\right) \psi\right)-\left(\delta_{q i} \omega^{n, q}-\sum_{j=1}^{q} w_{n, j} \ell_{n, i}^{\prime}\left(t^{n, j}\right) \omega^{n, j}-\ell_{n, i}\left(t^{n}\right) \omega^{n}, \psi\right) \\
& \quad-\mathrm{i} k_{n} w_{i}\left(\nabla u^{n, i}, \nabla \psi\right)+\mathrm{i} \lambda \int_{I_{n}} \ell_{n, i}(f(W), \psi) d t \\
& =\left(E^{n}, \ell_{n, i}\left(t^{n}\right) \psi\right)+\left(\Theta^{n, i}+A^{n, i}+B^{n, i}, \psi\right)-\left(J\left[\eta^{n}\right], \ell_{n, i}\left(t^{n}\right) \psi\right) \\
& \quad+\mathrm{i} \lambda \int_{I_{n}} \ell_{n, i}(f(W)-f(u), \psi) d t, \quad i=1, \ldots, q,
\end{aligned}
$$

where for $\eta^{n, j}=\eta\left(\cdot, t^{n, j}\right)$,

$$
\begin{aligned}
\Theta^{n, i} & :=\delta_{q i} \eta^{n, q}-\sum_{j=1}^{q} w_{n, j} \ell_{n, i}^{\prime}\left(t^{n, j}\right) \eta^{n, j}-\ell_{n, i}\left(t^{n}\right) \eta^{n+}, \\
A^{n, i} & :=\sum_{j=1}^{q} w_{n, j} \ell_{n, i}^{\prime} u^{n, j}-\int_{I_{n}} \ell_{n, i}^{\prime} u d t, \\
B^{n, i} & :=\mathrm{i} k_{n} w_{i} \Delta u^{n, i}-\mathrm{i} \int_{I_{n}} \ell_{n, i} \Delta u d t \\
J\left[\eta^{n}\right] & :=\eta^{n}-\eta^{n+}=\omega^{n+}-\omega^{n}=\left(P_{E}^{n}-P_{E}^{n-1}\right) u\left(t^{n}\right) .
\end{aligned}
$$

Here we used the definition of $W$ and $P_{E}^{n}$, and the fact that for any $\phi \in V_{h k}$ there holds

$$
\left(u^{n+1}, \phi^{n+1}\right)-\int_{I_{n}}\left(u, \phi_{t}\right) d t-\left(u^{n}, \phi^{n+}\right)+\mathrm{i} \int_{I_{n}}(\nabla u, \nabla \phi) d t-\mathrm{i} \lambda \int_{I_{n}}(f(u), \phi) d t=0 .
$$

In the convergence proof we will need a bound for $\widetilde{E}^{n, j}=\tau_{j}^{-1 / 2} E^{n, j}$, cf. (2.11). We write therefore the analog of (3.9) for $\widetilde{E}^{n, j}$ (recall that $E=\sum_{j=1}^{q} \tau_{j}^{1 / 2} \ell_{n, j} \widetilde{E}^{n, j}$ 
in $\left.I_{n}\right)$ :

$$
\begin{aligned}
\delta_{q i}\left(\widetilde{E}^{n, q}, \psi\right)-\sum_{j=1}^{q} & w_{n, j} \ell_{n, i}^{\prime}\left(t^{n, j}\right) \tau_{j}^{1 / 2} \tau_{i}^{-1 / 2}\left(\widetilde{E}^{n, j}, \psi\right)+\mathrm{i} k_{n} w_{i}\left(\nabla \widetilde{E}^{n, i}, \nabla \psi\right) \\
& -\mathrm{i} \lambda \tau_{i}^{-1 / 2} \int_{I_{n}} \ell_{n, i}(f(U)-f(W), \psi) d t \\
3.11)=\tau_{i}^{-1 / 2} & \left\{\left(E^{n}, \ell_{n, i}\left(t^{n}\right) \psi\right)+\left(\Theta^{n, i}+A^{n, i}+B^{n, i}, \psi\right)-\left(J\left[\eta^{n}\right], \ell_{n, i}\left(t^{n}\right) \psi\right)\right. \\
+ & \left.\mathrm{i} \lambda \int_{I_{n}} \ell_{n, i}(f(W)-f(u), \psi) d t\right\}, \quad i=1, \ldots, q .
\end{aligned}
$$

Our next task is to bound the terms $\Theta^{n, i}, A^{n, i}$ and $B^{n, i}$. In fact, in the proof of the following lemma, we derive appropriate estimates for these terms, which allow us to obtain the optimal-order convergence result of our scheme.

Lemma 3.1. For any $n, 0 \leq n \leq N-1$, and for $i=1, \ldots, q$ there holds

$$
\begin{aligned}
& \left\|\Theta^{n, i}\right\| \leq c k_{n}^{1 / 2}\left(\int_{I_{n}}\left\|h_{n}^{r} u_{t}\right\|_{r, h}^{2}\right)^{\frac{1}{2}}, \\
& \left\|A^{n, i}\right\| \leq c k_{n}^{q+1 / 2}\left\|u^{(q+1)}\right\|_{n}
\end{aligned}
$$

and

$$
\left\|B^{n, i}\right\| \leq c k_{n}^{q+1 / 2}\left\|\Delta u^{(q)}\right\|_{n},
$$

where $\Theta^{n, i}, A^{n, i}$ and $B^{n, i}$ are defined in (3.10).

Proof. For the estimate of $\Theta^{n, i}$ we note first that for $i=1, \ldots, q$,

$$
\delta_{q i}-\sum_{j=1}^{q} w_{n, j} \ell_{n, i}^{\prime}\left(t^{n, j}\right)-\ell_{n, i}\left(t^{n}\right)=\delta_{q i}-\int_{t^{n}}^{t^{n+1}} \ell_{n, i}^{\prime}(t) d t-\ell_{n, i}\left(t^{n}\right)=0 .
$$

This implies that there exist constants $\beta_{i j}$ (independent of $n$ ), such that

$$
\begin{aligned}
\Theta^{n, i} & =\delta_{q i} \eta^{n, q}-\sum_{j=1}^{q} w_{j} \ell_{i}^{\prime}\left(\tau_{j}\right) \eta^{n, j}-\ell_{i}(0) \eta^{n+} \\
& =\sum_{j=1}^{q} \beta_{i j}\left(\eta^{n, j}-\eta^{n, j-1}\right) \quad\left(\eta^{n, 0}:=\eta^{n+}\right) \\
& =\sum_{j=1}^{q} \beta_{i j} \int_{t^{n, j-1}}^{t^{n, j}} \eta_{t}(s) d s, \quad\left(t^{n, 0}:=t^{n}\right) .
\end{aligned}
$$

Since $\eta_{t}=\left(I-P_{E}^{n}\right) u_{t},(3.7)$ and (3.4b) imply

$$
\left\|\Theta^{n, i}\right\| \leq c \int_{I_{n}}\left\|h_{n}^{r} u_{t}\right\|_{r, h} \leq c k_{n}^{1 / 2}\left(\int_{I_{n}}\left\|h_{n}^{r} u_{t}\right\|_{r, h}^{2}\right)^{\frac{1}{2}} .
$$

Now let $\mathcal{I}_{q}^{n}$ be the interpolation operator at the $q+1$ points of $I_{n}$, consisting of the $q$ Radau points and the point $t_{n}$, i.e., $\mathcal{I}_{q}^{n}: C\left(\bar{I}_{n}\right) \rightarrow \mathbb{P}_{q}\left(\bar{I}_{n}\right)$ is such that

$$
\begin{aligned}
& \mathcal{I}_{q}^{n} y\left(t^{n, j}\right)=y\left(t^{n, j}\right), \quad j=1, \ldots, q, \\
& \mathcal{I}_{q}^{n} y\left(t^{n}\right)=y\left(t^{n+}\right) .
\end{aligned}
$$


Using this notation and the fact that, for each $x \in \Omega, \ell_{n, i}^{\prime} \mathcal{I}_{q}^{n} u$ is a polynomial of degree $2 q-2$ in $t$, we obtain

$$
\begin{aligned}
A^{n, i} & =\sum_{j=1}^{q} w_{n, j} \ell_{n, i}^{\prime}\left(t^{n, j}\right) \mathcal{I}_{q}^{n} u\left(t^{n, j}\right)-\int_{I_{n}} \ell_{n, i}^{\prime} u d t \\
& =\int_{I_{n}} \ell_{n, i}^{\prime}\left(\mathcal{I}_{q}^{n} u-u\right) d t .
\end{aligned}
$$

It follows from the approximating properties of the operator $\mathcal{I}_{q}^{n}$ that

$$
\begin{aligned}
\left\|A^{n, i}\right\| & \leq c\left(\int_{I_{n}}\left|\ell_{n, i}^{\prime}(t)\right|^{2} d t\right)^{\frac{1}{2}}\left\|\mathcal{I}_{q}^{n} u-u\right\|_{n} \\
& \leq c\left(k_{n}^{-1} \int_{0}^{1}\left|\ell_{i}^{\prime}(\tau)\right|^{2} d \tau\right)^{\frac{1}{2}} c k_{n}^{q+1}\left\|u^{(q+1)}\right\|_{n} \\
& \leq c k_{n}^{q+1 / 2}\left\|u^{(q+1)}\right\|_{n} .
\end{aligned}
$$

Analogously, using the fact that $\ell_{n, i} \mathcal{I}_{q-1}^{n} u$ is a polynomial of degree $2 q-2$ in $t$,

$$
\begin{aligned}
B^{n, i} & =\mathrm{i} \sum_{j=1}^{q} w_{n, j} \ell_{n, i}\left(t^{n, j}\right) \Delta u^{n, j}-\mathrm{i} \int_{I_{n}} \ell_{n, i} \Delta u d t \\
& =\mathrm{i} \int_{I_{n}} \ell_{n, i}\left(\mathcal{I}_{q-1}^{n}-I\right) \Delta u d t .
\end{aligned}
$$

Therefore,

$$
\begin{aligned}
\left\|B^{n, i}\right\| & \leq c\left(\int_{I_{n}}\left|\ell_{n, i}(t)\right|^{2} d t\right)^{\frac{1}{2}}\left\|\mathcal{I}_{q-1}^{n} \Delta u-\Delta u\right\|_{n} \\
& \leq c\left(k_{n} \int_{0}^{1}\left|\ell_{i}(\tau)\right|^{2} d \tau\right)^{\frac{1}{2}} c k_{n}^{q}\left\|\Delta u^{(q)}\right\|_{n} \\
& \leq c k_{n}^{q+1 / 2}\left\|\Delta u^{(q)}\right\|_{n},
\end{aligned}
$$

which completes the proof.

We now set $\psi=E^{n, i}$ in the i-th equation of (3.9) and sum from $i=1$ to $q$ and take real parts. We then have, since the left hand side of the resulting equation will be equal to the left hand side of (3.6) for $\varphi=E$, cf. (3.8b),

$$
\begin{aligned}
\frac{1}{2}\left\|E^{n+1}\right\|^{2}+ & \frac{1}{2}\left\|E^{n+}\right\|^{2}=\operatorname{Re}\left(E^{n}, E^{n+}\right)-\operatorname{Im} \lambda \int_{I_{n}}(f(U)-f(W), E) d t \\
& +\operatorname{Im} \lambda \int_{I_{n}}(f(u)-f(W), E) d t \\
& +\operatorname{Re} \sum_{i=1}^{q}\left(\Theta^{n, i}+A^{n, i}+B^{n, i}, E^{n, i}\right)-\operatorname{Re}\left(J\left[\eta^{n}\right], E^{n+}\right) .
\end{aligned}
$$

Using the properties of $f$, cf. Lemma 4.1, and (3.5a) we obtain

$$
\begin{aligned}
& \left|\int_{I_{n}}(f(U)-f(W), E) d t\right| \leq c\|E\|_{n}^{2}, \\
& \left|\int_{I_{n}}(f(u)-f(W), E) d t\right| \leq c\left\{k_{n}^{q}\left\|u^{(q)}\right\|_{n}+k_{n}^{1 / 2} \max _{I_{n}}\left\|h_{n}^{r} u\right\|_{r, h}\right\}\|E\|_{n} .
\end{aligned}
$$


Using the fact that,

$$
\|E\|_{n}=\left(\sum_{j=1}^{q} w_{n, j}\left\|E^{n, j}\right\|^{2}\right)^{\frac{1}{2}}=k_{n}^{1 / 2}\left(\sum_{j=1}^{q} w_{j}\left\|E^{n, j}\right\|^{2}\right)^{\frac{1}{2}},
$$

Lemma 3.1, (3.18) and the observation that $J\left[\eta^{n}\right]=0$ if $S_{h}^{n}=S_{h}^{n-1}$, from (3.17) we obtain

$$
\begin{aligned}
\left\|E^{n+1}\right\|^{2} \leq(1 & \left.+\beta_{n}\right)\left\|E^{n}\right\|^{2}+c\|E\|_{n}^{2} \\
& +c k_{n}^{2 q}\left(\left\|u^{(q)}\right\|_{n}^{2}+\left\|u^{(q+1)}\right\|_{n}^{2}+\left\|\Delta u^{(q)}\right\|_{n}^{2}\right) \\
& +c \int_{I_{n}}\left\|h_{n}^{r} u_{t}\right\|_{r, h}^{2} d t+c k_{n} \max _{I_{n}}\left\|h_{n}^{r} u\right\|_{r, h}^{2} \\
& +M_{n}\left\|J\left[\eta^{n}\right]\right\|^{2},
\end{aligned}
$$

where $M_{n}$ is a number depending on $n$ which will be specified in the sequel and

$$
\beta_{n}= \begin{cases}0 & \text { if } S_{h}^{n}=S_{h}^{n-1}, \quad n=1, \ldots, N-1 . \\ \frac{1}{M_{n}-1} & \text { otherwise, }\end{cases}
$$

An essential step now is the estimation of $\|E\|_{n}$ in terms of $\left\|E^{n}\right\|$ and of consistency terms. Indeed, we have the following result:

Lemma 3.2. For any $n, 0 \leq n \leq N-1$, and $k_{n}$ sufficiently small, there holds

$$
\begin{aligned}
\|E\|_{n}^{2} \leq c k_{n} & \left\{\left\|E^{n}\right\|^{2}+k_{n}^{2 q+1}\left(\left\|u^{(q)}\right\|_{n}^{2}+\left\|u^{(q+1)}\right\|_{n}^{2}+\left\|\Delta u^{(q)}\right\|_{n}^{2}\right)\right. \\
+ & \left.k_{n}\left(\int_{I_{n}}\left\|h_{n}^{r} u_{t}\right\|_{r, h}^{2} d t+k_{n} \max _{I_{n}}\left\|h_{n}^{r} u\right\|_{r, h}^{2}\right)+\left\|J\left[\eta^{n}\right]\right\|^{2}\right\} .
\end{aligned}
$$

Proof. The proof is based on the representation of $E$ as $E=\sum_{j=1}^{q} \tau_{j}^{1 / 2} \ell_{n, j} \widetilde{E}^{n, j}$ in $I_{n}$, and on the observation of Lemma 2.1. To this end, let $\psi=\widetilde{E}^{n, i}$ in (3.11) and sum from $i=1$ to $q$ and take real parts. Since

$$
\sum_{i=1}^{q} \delta_{q i}\left(\widetilde{E}^{n, q}, \widetilde{E}^{n, i}\right)-\sum_{i, j=1}^{q} w_{n, j} \ell_{n, i}^{\prime}\left(t^{n, j}\right) \tau_{j}^{1 / 2} \tau_{i}^{-1 / 2}\left(\widetilde{E}^{n, j}, \widetilde{E}^{n, i}\right)=\left(\left(\widetilde{\mathcal{M}} \widetilde{\mathbf{E}}^{n}, \widetilde{\mathbf{E}}^{n}\right)\right),
$$

where $\widetilde{\mathcal{M}}$ is defined in $(2.7)$ and $\widetilde{\mathbf{E}}^{n} \in\left(S_{h}^{n}\right)^{q}, \widetilde{\mathbf{E}}^{n}=\left(\widetilde{E}^{n, 1}, \ldots, \widetilde{E}^{n, q}\right)$, (here we use the notation introduced in the proof of Theorem 2.1) by Lemma 2.1 we obtain

$$
\operatorname{Re}\left(\left(\widetilde{\mathcal{M}} \widetilde{\mathbf{E}}^{n}, \widetilde{\mathbf{E}}^{n}\right)\right) \geq \alpha \sum_{j=1}^{q}\left\|\widetilde{E}^{n, j}\right\|^{2} .
$$

Using (4.3) and the fact that $\int_{I_{n}} \ell_{n, i}^{2}(t) d t=w_{i} k_{n}$, we obtain

$$
\left|\sum_{i=1}^{q} \lambda \tau_{i}^{-1 / 2} \int_{I_{n}} \ell_{n, i}\left(f(U)-f(W), \widetilde{E}^{n, i}\right) d t\right| \leq c k_{n}^{1 / 2}\left\{\sum_{i=1}^{q}\left\|\widetilde{E}^{n, i}\right\|^{2}\right\}^{1 / 2}\|E\|_{n} .
$$

Applying a similar treatment to the last term in (3.11), we conclude that

$$
\begin{aligned}
\alpha \sum_{j=1}^{q}\left\|\widetilde{E}^{n, j}\right\|^{2} \leq & c\left\{\sum_{j=1}^{q}\left\|\widetilde{E}^{n, j}\right\|^{2}\right\}^{1 / 2}\left\{\left\|E^{n}\right\|+c k_{n}^{1 / 2}\|E\|_{n}+c k_{n}^{1 / 2}\|u-W\|_{n}\right. \\
& \left.+\left(\sum_{i=1}^{q}\left[\left\|\Theta^{n, i}\right\|^{2}+\left\|A^{n, i}\right\|^{2}+\left\|B^{n, i}\right\|^{2}\right]\right)^{1 / 2}+\left\|J\left[\eta^{n}\right]\right\|\right\} .
\end{aligned}
$$


Using the analog of (3.19) for $\widetilde{E}^{n, i}$, and taking $k_{n}$ sufficiently small we obtain

$$
\begin{aligned}
\|E\|_{n}^{2} \leq & c k_{n}\left\|E^{n}\right\|^{2}+c k_{n}^{2}\|u-W\|_{n}^{2} \\
& +c k_{n}\left(\sum_{i=1}^{q}\left[\left\|\Theta^{n, i}\right\|^{2}+\left\|A^{n, i}\right\|^{2}+\left\|B^{n, i}\right\|^{2}\right]\right)+c k_{n}\left\|J\left[\eta^{n}\right]\right\|^{2},
\end{aligned}
$$

the desired result now follows in view of (3.5a) and Lemma 3.1.

We are now ready to prove the main convergence result for the modified scheme.

Theorem 3.1. Let $u$ be the solution of (1.1). If $U$ is the solution of (1.3), then it satisfies

$$
\begin{gathered}
\max _{I_{n}}\|E\| \leq C_{n}\left\|h_{0}^{r} u^{0}\right\|_{r, h}+C_{n}\left\{\sum _ { m = 0 } ^ { n } \left(k_{m}^{2 q}\left(\left\|u^{(q)}\right\|_{m}^{2}+\left\|u^{(q+1)}\right\|_{m}^{2}+\left\|\Delta u^{(q)}\right\|_{m}^{2}\right)\right.\right. \\
\left.\left.+c \int_{I_{m}}\left\|h_{m}^{r} u_{t}\right\|_{r, h}^{2} d t+c k_{m} \max _{I_{m}}\left\|h_{m}^{r} u\right\|_{r, h}^{2}\right)\right\}^{1 / 2} \\
+c C_{n} \mathcal{N}_{C} \max _{1 \leq m \leq n}\left\|J\left[\eta^{m}\right]\right\|, \quad n=0,1, \ldots, N-1,
\end{gathered}
$$

where $C_{n}=c e^{c t_{n}}$, and therefore

$$
\begin{gathered}
\max _{t \in[0, T]}\|u(t)-U(t)\| \leq C e^{c T}\left\{\max _{m} k_{m}^{q} \max _{I_{m}}\left(\left\|u^{(q)}\right\|+\left\|u^{(q+1)}\right\|+\left\|\Delta u^{(q)}\right\|\right)\right. \\
+\max _{m} \max _{I_{m}}\left(\left\|h_{m}^{r} u_{t}\right\|_{r, h}+\left\|h_{m}^{r} u\right\|_{r, h}\right) \\
\left.+\mathcal{N}_{C} \max _{m}\left\|J\left[\eta^{m}\right]\right\|\right\} .
\end{gathered}
$$

Proof. In view of Lemma 3.2, inequality (3.20) implies

$$
\begin{aligned}
\left\|E^{n+1}\right\|^{2} \leq(1 & \left.+\beta_{n}+c k_{n}\right)\left\|E^{n}\right\|^{2} \\
& +c k_{n}^{2 q}\left(\left\|u^{(q)}\right\|_{n}^{2}+\left\|u^{(q+1)}\right\|_{n}^{2}+\left\|\Delta u^{(q)}\right\|_{n}^{2}\right) \\
& +c \int_{I_{n}}\left\|h_{n}^{r} u_{t}\right\|_{r, h}^{2} d t+c k_{n} \max _{I_{n}}\left\|h_{n}^{r} u\right\|_{r, h}^{2} \\
& +\left(c k_{n}+M_{n}\right)\left\|J\left[\eta^{n}\right]\right\|^{2} .
\end{aligned}
$$

Therefore,

$$
\begin{aligned}
\left\|E^{n+1}\right\|^{2} \leq & \prod_{j=0}^{n}\left(1+\beta_{j}+c k_{j}\right)\left\|E^{0}\right\|^{2} \\
(3.25)+ & +c \sum_{m=0}^{n}\left(\prod_{j=m+1}^{n}\left(1+\beta_{j}+c k_{j}\right)\right)\left(k_{m}^{2 q}\left\{\left\|u^{(q)}\right\|_{m}^{2}+\left\|u^{(q+1)}\right\|_{m}^{2}+\left\|\Delta u^{(q)}\right\|_{m}^{2}\right\}\right. \\
& \left.+c \int_{I_{m}}\left\|h_{m}^{r} u_{t}\right\|_{r, h}^{2} d t+c k_{m} \max _{I_{m}}\left\|h_{m}^{r} u\right\|_{r, h}^{2}+\left(c k_{m}+M_{m}\right)\left\|J\left[\eta^{m}\right]\right\|^{2}\right) .
\end{aligned}
$$

Now, fix $n$ and choose $M_{m}=M=\mathcal{N}_{C}(n), m=1, \ldots, n$, where $\mathcal{N}_{C}(n)$ is the number of times where $S_{h}^{j} \neq S_{h}^{j-1}, j=1, \ldots n$ (in the case where $\mathcal{N}_{C}(n)=0$ or 1 
we take $M=2)$. Then, $\beta_{j}=\beta=\frac{1}{M-1}$, for $S_{h}^{j} \neq S_{h}^{j-1}$, cf. (3.21),

$$
\begin{aligned}
\prod_{j=0}^{n}\left(1+\beta_{j}+c k_{j}\right) & \leq \prod_{j=0}^{n}\left(1+2 c k_{j}\right) \prod_{\substack{j=0 \\
\beta \geq c k_{j}}}^{n}(1+2 \beta) \\
& \leq \prod_{j=0}^{n}\left(1+2 c k_{j}\right)(1+2 \beta)^{M} \leq e^{2 c t_{n+1}} 3 e^{2} .
\end{aligned}
$$

Set now $C_{n}:=\left(3 e^{2 c t_{n}+2}\right)^{1 / 2}$. Then since $E^{0}=u^{0}-P_{E}^{0} u^{0}, J\left[\eta^{0}\right]=0,\left(k_{m} \leq M\right)$

$$
\begin{aligned}
\left\|E^{n+1}\right\| \leq & C_{n+1}\left\|u^{0}-P_{E}^{0} u^{0}\right\| \\
+ & c C_{n+1}\left\{\sum _ { m = 0 } ^ { n } \left(k_{m}^{2 q}\left(\left\|u^{(q)}\right\|_{m}^{2}+\left\|u^{(q+1)}\right\|_{m}^{2}+\left\|\Delta u^{(q)}\right\|_{m}^{2}\right)\right.\right. \\
& \left.\left.+\int_{I_{m}}\left\|h_{m}^{r} u_{t}\right\|_{r, h}^{2} d t+k_{m} \max _{I_{m}}\left\|h_{m}^{r} u\right\|_{r, h}^{2}\right)\right\}^{1 / 2} \\
& +c C_{n+1} \sqrt{M}\left(\sum_{m=1}^{n}\left\|J\left[\eta^{m}\right]\right\|^{2}\right)^{1 / 2}
\end{aligned}
$$

where $M=\mathcal{N}_{C}(n)$. In view of Lemma 3.2 and of (3.4b), we obtain for any $n=$ $0, \ldots, N-1$,

$$
\begin{aligned}
\|E\|_{n} \leq & k_{n}^{1 / 2} c C_{n}\left\|h_{0}^{r} u^{0}\right\|_{r, h} \\
+ & k_{n}^{1 / 2} c C_{n}\left\{\sum _ { m = 0 } ^ { n } \left(k_{m}^{2 q}\left(\left\|u^{(q)}\right\|_{m}^{2}+\left\|u^{(q+1)}\right\|_{m}^{2}+\left\|\Delta u^{(q)}\right\|_{m}^{2}\right)\right.\right. \\
& \left.\left.+\int_{I_{m}}\left\|h_{m}^{r} u_{t}\right\|_{r, h}^{2} d t+k_{m} \max _{I_{m}}\left\|h_{m}^{r} u\right\|_{r, h}^{2}\right)\right\}^{1 / 2} \\
& +k_{n}^{1 / 2} c C_{n} \sqrt{\mathcal{N}_{C}(n-1)}\left(\sum_{m=1}^{n}\left\|J\left[\eta^{m}\right]\right\|^{2}\right)^{1 / 2}
\end{aligned}
$$

Since $\left.E\right|_{I_{n}} \in V_{h k}^{n}$, the inverse inequality

$$
\max _{I_{n}}|y(t)| \leq c_{I} k_{n}^{-1 / 2}\left(\int_{I_{n}}|y(t)|^{2} d t\right)^{1 / 2}, \quad \forall y \in \mathbb{P}_{q-1}\left(I_{n}\right), \quad c_{I}>0,
$$

implies

$$
\begin{aligned}
\max _{I_{n}}\|E\| \leq & c C_{n}\left\|h_{0}^{r} u^{0}\right\|_{r, h} \\
+ & c C_{n}\left\{\sum _ { m = 0 } ^ { n } \left(k_{m}^{2 q}\left(\left\|u^{(q)}\right\|_{m}^{2}+\left\|u^{(q+1)}\right\|_{m}^{2}+\left\|\Delta u^{(q)}\right\|_{m}^{2}\right)\right.\right. \\
& \left.\left.+\int_{I_{m}}\left\|h_{m}^{r} u_{t}\right\|_{r, h}^{2} d t+k_{m} \max _{I_{m}}\left\|h_{m}^{r} u\right\|_{r, h}^{2}\right)\right\}^{1 / 2} \\
& +c C_{n} \sqrt{\mathcal{N}_{C}}\left(\sum_{m=1}^{n}\left\|J\left[\eta^{m}\right]\right\|^{2}\right)^{1 / 2}
\end{aligned}
$$

and (3.23) follows. 


\section{Convergence of the nOnlinear scheme}

In this section we shall use the results of Section 3 for the modified scheme to show the convergence of (1.2) to the solution of the nonlinear Schrödinger equation (1.1). First we make a concrete choice of the function $f$ used in the modified scheme which satisfies certain properties, some of them already used in the previous sections:

Lemma 4.1. Let $M:=\sup _{[0, T]}\|u\|_{\infty}+1$. Then, there exists a function $f: \mathbb{C} \rightarrow \mathbb{C}$ such that

$$
\begin{aligned}
& f(z)=|z|^{2} z, \quad \text { if }|z| \leq M, \\
& |f(z)| \leq c_{1}|z|, \quad c_{1}>0, \quad \forall z \in \mathbb{C}, \\
& |f(z)-f(w)| \leq c_{2}|z-w|, \quad c_{2}>0, \quad \forall z, w \in \mathbb{C} \\
& \left\|\nabla_{x}\left(f\left(v_{1}\right)-f\left(v_{2}\right)\right)\right\| \leq c_{3}\left\|\nabla_{x}\left(v_{1}-v_{2}\right)\right\|, \quad c_{3}=c_{3}\left(M^{\prime}\right)>0, \\
& \forall v_{1}, v_{2} \in H_{0}^{1} \text { with }\left\|v_{1}\right\|_{1, \infty} \leq M^{\prime} .
\end{aligned}
$$

Proof . Let $\gamma:[0, \infty) \rightarrow \mathbb{R}$ be a $C^{2}$ function with bounded derivatives up to second order, such that

$$
\gamma(s)=\left\{\begin{array}{ll}
s, & s \leq M^{2} \\
(2 M)^{2}, & s \geq(2 M)^{2}
\end{array} \quad \text { and } M^{2} \leq \gamma(s) \leq(2 M)^{2}, \quad \text { for } M^{2} \leq s \leq(2 M)^{2}\right.
$$

Define $f: \mathbb{C} \rightarrow \mathbb{C}$, by $f(z)=\gamma\left(|z|^{2}\right) z$. Then (4.1), (4.2) hold. Also, since

$$
f(z)-f(w)=\gamma\left(|z|^{2}\right)(z-w)+\left[\gamma\left(|z|^{2}\right)-\gamma\left(|w|^{2}\right)\right] w
$$

it is a simple matter to see that (4.3) holds in the cases $|z|,|w|>4 M$ or $|z|,|w|<$ $4 M$, by using $\left[\gamma\left(|z|^{2}\right)-\gamma\left(|w|^{2}\right)\right]=\gamma^{\prime}(\xi)(|z|-|w|)(|z|+|w|)$. Similarly, if $|z|>$ $4 M, 2 M \leq|w| \leq 4 M$. If $|w| \leq 2 M$, (4.3) follows by (4.5) since $|w-z| \geq 2 M$ and hence $|w-z| \geq|w|$. Relation (4.4) is proved using similar arguments.

By Lemma 4.1 it is clear that if we show that the solution of (1.3) satisfies

$$
\max _{[0, T]}\|U\|_{\infty} \leq M
$$

then $U$ will be a solution of (1.2) as well and the estimate of Theorem 3.1 will remain valid. By an inverse inequality, the proof of (4.6) rests (up to a logarithmic factor) on the control of $\max _{[0, T]}\|\nabla U\|$. In the rest of this section we shall see that $\|\nabla U\|$ can be controlled under some assumptions on the structure of the mesh. We begin with

Proposition 4.1. For a given $n, 1 \leq n \leq N-1$, assume that the following hold:

$$
\begin{aligned}
& S_{h}^{n-1} \subset S_{h}^{n}, \\
& \left\|\nabla P^{n} v\right\| \leq C_{P}\|\nabla v\|, \quad \forall v \in H_{0}^{1}(\Omega),
\end{aligned}
$$


where $P^{n}$ is the $L^{2}$-projection onto $S_{h}^{n}$. Then

$$
\begin{aligned}
\left\|\nabla E^{n+1}\right\|^{2} \leq(1 & \left.+c k_{n}\right)\left\|\nabla E^{n}\right\|^{2} \\
& +c k_{n}^{2(q-1)}\left(\left\|\nabla u^{(q-1)}\right\|_{n}^{2}+\left\|\nabla u^{(q)}\right\|_{n}^{2}+\left\|\Delta u^{(q-1)}\right\|_{n}^{2}\right) \\
& +c \int_{I_{n}}\left\|h_{n}^{r-1} u_{t}\right\|_{r, h}^{2} d t+c k_{n} \max _{I_{n}}\left\|h_{n}^{r-1} u\right\|_{r, h}^{2} \\
& +\left(1+c k_{n}\right)\left\|\nabla J\left[\eta^{n}\right]\right\|^{2} .
\end{aligned}
$$

Remark 4.1. The bound (4.8) was proved in Crouzeix and Thomée [CrT] in one and two dimensions, under some restrictions on the variation of the mesh, but without the assumption of quasiuniformity, cf. [CrT] for details.

Proof. We introduce first the discrete operator $A_{h}^{n}: H_{0}^{1}(\Omega) \rightarrow S_{h}^{n}$, defined by

$$
\left(A_{h}^{n} v, \chi\right)=(\nabla v, \nabla \chi), \quad \forall \chi \in S_{h}^{n}
$$

Next, we set $\psi=A_{h}^{n} E^{n, i}$ in (3.9), sum over $i$, from $i=1$ to $q$, and take real parts. Then, since the left hand side of the resulting equation is equal to the left hand side of (3.6) for $\varphi=A_{h}^{n} E \in V_{h k}^{n}$, we obtain by using $S_{h}^{n-1} \subset S_{h}^{n}$,

$$
\begin{aligned}
\frac{1}{2}\left\|\nabla E^{n+1}\right\|^{2}+ & \frac{1}{2}\left\|\nabla E^{n+}\right\|^{2}=\operatorname{Re}\left(\nabla E^{n}, \nabla E^{n+}\right) \\
& -\operatorname{Im} \lambda \int_{I_{n}}\left(\nabla P^{n}(f(U)-f(W))-\nabla P^{n}(f(u)-f(W)), \nabla E\right) d t \\
& +\operatorname{Re} \sum_{i=1}^{q}\left(\nabla P^{n}\left[\Theta^{n, i}+A^{n, i}+B^{n, i}\right], \nabla E^{n, i}\right)-\operatorname{Re}\left(J\left[\eta^{n}\right], A_{h}^{n} E^{n+}\right) .
\end{aligned}
$$

We estimate the terms in the right hand side of (4.10) in the following lemma whose proof is given below.

Lemma 4.2. For any $n, 0 \leq n \leq N-1$, there hold

$$
\begin{gathered}
\left|\int_{I_{n}}\left(\nabla P^{n}(f(U)-f(W))-\nabla P^{n}(f(u)-f(W)), \nabla E\right) d t\right| \leq c\|\nabla E\|_{n}^{2} \\
+c k_{n}^{2 q}\left\|\nabla u^{(q)}\right\|_{n}^{2}+c k_{n} \max _{I_{n}}\left\|h_{n}^{r-1} u\right\|_{r, h}^{2}
\end{gathered}
$$

and

$$
\begin{aligned}
\mid \sum_{i=1}^{q}\left(\nabla P ^ { n } \left[\Theta^{n, i}+A^{n, i}\right.\right. & \left.\left.+B^{n, i}\right], \nabla E^{n, i}\right) \mid \leq c\|\nabla E\|_{n}^{2}+c \int_{I_{n}}\left\|h_{n}^{r-1} u\right\|_{r, h}^{2} \\
& +c k_{n}^{2(q-1)}\left(\left\|\nabla u^{(q)}\right\|_{n}^{2}+\int_{I_{n}}\left\|\Delta u^{(q-1)}\right\|_{1}^{2} d t\right) .
\end{aligned}
$$

Since $S_{h}^{n-1} \subset S_{h}^{n}$, we have $\left(\nabla J\left[\eta^{n}\right], \nabla \psi\right)=-\left(\nabla J\left[\omega^{n}\right], \nabla \psi\right)=0$, for all $\psi \in S_{h}^{n-1}$. Therefore

$$
\left(J\left[\eta^{n}\right], A_{h}^{n} E^{n+}\right)=\left(\nabla J\left[\eta^{n}\right], \nabla E^{n+}\right)=\left(\nabla J\left[\eta^{n}\right], \nabla\left(E^{n+}-E^{n}\right)\right) .
$$


On the other hand, $\frac{1}{2}\left\|\nabla E^{n+}\right\|^{2}-\operatorname{Re}\left(\nabla E^{n}, \nabla E^{n+}\right)=\frac{1}{2}\left\|\nabla\left(E^{n+}-E^{n}\right)\right\|^{2}-\frac{1}{2}\left\|\nabla E^{n}\right\|^{2}$. Hence, using the last relation, (4.11) and Lemma 4.2 in (4.10) one finally obtains

$$
\begin{aligned}
\left\|\nabla E^{n+1}\right\|^{2} \leq \| & \nabla E^{n}\left\|^{2}+c\right\| \nabla E \|_{n}^{2} \\
& +c k_{n}^{2(q-1)}\left(\left\|\nabla u^{(q)}\right\|_{n}^{2}+\int_{I_{n}}\left\|\Delta u^{(q-1)}\right\|_{1}^{2} d t\right) \\
& +c \int_{I_{n}}\left\|h_{n}^{r-1} u_{t}\right\|_{r, h}^{2} d t+c k_{n} \max _{I_{n}}\left\|h_{n}^{r-1} u\right\|_{r, h}^{2} \\
& +\left\|\nabla J\left[\eta^{n}\right]\right\|^{2} .
\end{aligned}
$$

Next, starting from the error equation (3.11) with $\psi=A_{h}^{n} \widetilde{E}^{n, i}$, and adapting the proof of Lemma 3.2 to the present case, we obtain

$$
\begin{gathered}
\|\nabla E\|_{n}^{2} \leq c k_{n}\left\{\left\|\nabla E^{n}\right\|^{2}+c k_{n}^{2 q-1}\left(\left\|\nabla u^{(q)}\right\|_{n}^{2}+\int_{I_{n}}\left\|\Delta u^{(q-1)}\right\|_{1}^{2} d t\right)\right. \\
+c k_{n}\left(\int_{I_{n}}\left\|h_{n}^{r-1} u_{t}\right\|_{r, h}^{2} d t+c k_{n} \max _{I_{n}}\left\|h_{n}^{r-1} u\right\|_{r, h}^{2}\right) \\
\left.+\left\|\nabla J\left[\eta^{n}\right]\right\|^{2}\right\} .
\end{gathered}
$$

Relations (4.12) and (4.13) complete the proof.

Proof of Lemma 4.2. Since $\sup _{[0, T]}\|W\|_{1, \infty}$ is bounded, cf. Remark 4.2, using (4.4) and (4.8) we obtain

$$
\left|\int_{I_{n}}\left(\nabla P^{n}(f(U)-f(W)), \nabla E\right) d t\right| \leq c\|\nabla E\|_{n}^{2} .
$$

Also, the definition and the approximation properties of $\mathcal{I}_{q-1}^{n}$ imply

$$
\begin{aligned}
\mid \int_{I_{n}}\left(\nabla P^{n}(f(u)-\right. & f(W)), \nabla E) d t \mid \leq c\left(\int_{I_{n}}\|\nabla(u-W)\|^{2} d t\right)^{1 / 2}\|\nabla E\|_{n} \\
& \leq c\left(\int_{I_{n}}\left\|\nabla u-\mathcal{I}_{q-1}^{n} \nabla u\right\|^{2}+\left\|\mathcal{I}_{q-1}^{n}(\nabla u-\nabla \omega)\right\|^{2} d t\right)^{1 / 2}\|\nabla E\|_{n} \\
& \leq c\left(k_{n}^{q}\left\|\nabla u^{(q)}\right\|_{n}+c k_{n}^{1 / 2} \max _{I_{n}}\left\|h_{n}^{r-1} u\right\|_{r, h}\right)\|\nabla E\|_{n} .
\end{aligned}
$$

Similar arguments to those used in the proof of (3.12a), (4.8) and the analog of (3.19) for $\nabla E$ give

$$
\left|\sum_{i=1}^{q}\left(\nabla P^{n} \Theta^{n, i}, \nabla E^{n, i}\right)\right| \leq c\left(\int_{I_{n}}\left\|h_{n}^{r-1} u_{t}\right\|_{r, h}^{2}\right)^{1 / 2}\|\nabla E\|_{n} .
$$

To estimate $A^{n, i}$, we note that $\ell_{n, i}^{\prime} \mathcal{I}_{q}^{n} \nabla P^{n} u$ is a polynomial of degree $2 q-2$ in $t$. Therefore

$$
\begin{aligned}
\nabla P^{n} A^{n, i} & =\sum_{j=1}^{q} w_{n, j} \ell_{n, i}^{\prime}\left(t^{n, j}\right) \mathcal{I}_{q}^{n} \nabla P^{n} u\left(t^{n, j}\right)-\int_{I_{n}} \ell_{n, i}^{\prime} \nabla P^{n} u d t \\
& =\int_{I_{n}} \ell_{n, i}^{\prime}\left(\mathcal{I}_{q}^{n} \nabla P^{n} u-\nabla P^{n} u\right) d t .
\end{aligned}
$$

Hence, cf. (3.15),

$$
\left\|\nabla P^{n} A^{n, i}\right\| \leq c k_{n}^{q-1 / 2}\left\|\nabla P^{n} u^{(q)}\right\|_{n} \leq c k_{n}^{q-1 / 2}\left\|\nabla u^{(q)}\right\|_{n} .
$$


Using similar arguments, see also (3.16), one finally obtains,

$$
\begin{aligned}
\left\|\nabla P^{n} B^{n, i}\right\| & \leq c k_{n}^{q-1 / 2}\left(\int_{I_{n}}\left\|\nabla P^{n} \Delta u^{(q-1)}\right\|^{2}\right)^{\frac{1}{2}} \\
& \leq c k_{n}^{q-1 / 2}\left(\int_{I_{n}}\left\|\Delta u^{(q-1)}\right\|_{1}^{2}\right)^{\frac{1}{2}},
\end{aligned}
$$

and the proof of the lemma is complete.

Remark 4.2. It is reasonable to assume that $\sup _{[0, T]}\|W\|_{1, \infty}$ is bounded by a constant independent of $h$, without the assumption of quasiuniformity, cf. [SW].

Before stating the main result of this section we recall that the quantities $k, h, \underline{h}$ and $C_{N}$ were defined in the introduction, cf. (1.5).

Theorem 4.1. Assume that for each $n, 1 \leq n \leq N-1$, there hold

$$
\begin{aligned}
& S_{h}^{n-1} \subset S_{h}^{n}, \\
& \left\|\nabla P^{n} v\right\| \leq C_{P}\|\nabla v\|, \quad \forall v \in H_{0}^{1}(\Omega), \quad \text { or } \quad k_{n} \geq C_{N}\left(k^{2 q}+h^{2 r}\right) .
\end{aligned}
$$

Then for $h$ and $k$ small enough the solution $U$ of (1.3) is a solution of (1.2) as well which satisfies the error bound of Theorem 3.1.

Proof. To show the desired result, it suffices to prove

$$
\max _{[0, T]}\|E\|_{\infty} \leq \beta(h, k, \underline{h}), \quad \beta(h, k, \underline{h}) \rightarrow 0 \quad \text { as } h, k \rightarrow 0 .
$$

To establish (4.14) we observe first that, [Th, p. 67],

$$
\max _{I_{n}}\|E\|_{\infty} \leq c|\ln (\underline{h})| \max _{I_{n}}\|\nabla E\| .
$$

To estimate $\max _{I_{n}}\|\nabla E\|$ we assume first that for all $n$ up to $n_{0}$ the hypotheses of Proposition 4.1 are valid $\left(S_{h}^{n-1} \subset S_{h}^{n}\right)$, then as in the proof of Theorem 3.1 one can show that for each $n, 0 \leq n \leq n_{0}$, there holds

$$
\begin{aligned}
\max _{I_{n}}\|\nabla E\| \leq & c C_{n}\left(\left\|h_{0}^{r-1} u^{0}\right\|_{r, h}\right. \\
+ & \left\{\sum _ { m = 0 } ^ { n } \left(k_{m}^{2 q-2}\left(\left\|\nabla u^{(q)}\right\|_{m}^{2}+\int_{I_{m}}\left\|\Delta u^{(q-1)}\right\|_{1}^{2}\right)\right.\right. \\
& \left.\left.\left.+c \int_{I_{m}}\left\|h_{m}^{r-1} u_{t}\right\|_{r, h}^{2} d t+c k_{m} \max _{I_{m}}\left\|h_{m}^{r-1} u\right\|_{r, h}\right)\right\}^{1 / 2}\right) \\
& +c C_{n} \sqrt{\mathcal{N}_{C}(n-1)} \max _{1 \leq m \leq n}\left\|J\left[\eta^{m}\right]\right\| .
\end{aligned}
$$

Hence up to $t^{n_{0}+1},(4.14)$ holds provided $\ln (\underline{h})\left(h^{r-1}+k^{q-1}\right) \rightarrow 0$ as $h, k \rightarrow 0$, cf. Remark 4.3. At the first time where $S_{h}^{n-1} \nsubseteq S_{h}^{n}$, say at $n_{0}+1$, set $\psi=E^{n, i}$ in (3.9) sum over $i$ and take imaginary parts. Then using the estimates of Lemma 3.1 and Theorem 3.1 we obtain

$$
\|\nabla E\|_{n_{0}+1} \leq c\left(k^{q}+h^{r}\right)+c \mathcal{N}_{C}\left(n_{0}\right) h^{r} .
$$

And the inverse inequality (3.28) implies

$$
\max _{I_{n_{0}+1}}\|\nabla E\| \leq c k_{n_{0}+1}^{-1 / 2}\left(k^{q}+h^{r}\right)+c k_{n_{0}+1}^{-1 / 2} \mathcal{N}_{C}\left(n_{0}\right) h^{r} .
$$


which in turn implies (4.14) for the interval $I_{n_{0}+1}$ in view of our assumption on the mesh in this case. Proceeding in time in a similar fashion we obtain the desired result.

Remark 4.3. For $q=1, \ln (\underline{h})\left(h^{r-1}+k^{q-1}\right)$ does not converge to zero. However we can redo the estimates of this section to obtain a rate of $O\left(k^{q}\right)$. Since this will require higher regularity, we reserve it only for the case $q=1$.

\section{ACKNOWLEDGEMENTS}

This work was supported in part by a research grant from the University of Crete and by the Mathematics Department of the University of Tennessee. The work of O. Karakashian was partly supported by NSF grant INT-9504845.

\section{REFERENCES}

[ADK] G.D. Akrivis, V.A. Dougalis and O. A. Karakashian, On fully discrete Galerkin methods of second-order temporal accuracy for the nonlinear Schrödinger equation, Numer. Math. 59 (1991), 31-53. MR 92a:65256

[BO] I. Babuška and J. E. Osborn, Analysis of finite element methods for second order boundary value problems using mesh dependent norms, Numer. Math. 34 (1980), 41-62. MR 81g:65143

[BL] L. Bales and I. Lasiecka, Continuous finite elements in space and time for the nonhomogeneous wave equation, Computers Math. Applic. 27 (1994), 91-102. MR 94k:65138

[BS] S. C. Brenner and L. R. Scott, The Mathematical Theory of Finite Element Methods, Springer -Verlag, New York, 1994. MR 95f:65001

[Br] F.E. Browder, Existence and uniqueness theorems for solutions of nonlinear boundary value problems, Applications of Partial Differential Equations (R. Finn, ed.), American Mathematical Society, Providence, pp. 24-29, 1965. MR 33:6092

[CGT] R.Y. Chiao, E. Garmire and C. Townes, Self-trapping of optical beams, Phys. Rev. Lett. 73 (1964), 479-482.

[CrT] M. Crouzeix and V. Thomée, The stability in $L_{p}$ and $W_{p}^{1}$ of the $L_{2}$-projection onto finite element spaces, Math. Comp. 48 (1987), 521-532. MR 88f:41016

[Ci] P.G. Ciarlet, The Finite Element Method for Elliptic Problems, North-Holland, Amsterdam, 1978. MR 58:25001

[DR] P.J. Davis and P. Rabinowitz, Methods of Numerical Integration, Academic Press, New York, 1975. MR 56:7119

[DV] K. Dekker and J.D. Verwer, Stability of Runge-Kutta Methods for Stiff Nonlinear Differential Equations, CWI Monographs, North-Holland, Amsterdam, 1984. MR 86g:65003

[Dr] W. Dörfler, A time- and space-adaptive algorithm for the linear time-dependent Schrödinger equation, Numer. Math. 73 (1996), 419-448. MR 97g:65183

[D] T. Dupont, Mesh modification for evolution equations, Math. Comp. 39 (1982), 85-107. MR 84g:65131

[EJ1] K. Eriksson and C. Johnson, Adaptive finite element methods for parabolic problems I: A linear model problem, SIAM J. Numer. Anal. 28 (1991), 43-77. MR 91m:65274

[EJ2] K. Eriksson and C. Johnson, Adaptive finite element methods for parabolic problems II: Optimal error estimates in $L_{\infty} L_{2}$ and $L_{\infty} L_{\infty}$, SIAM J. Numer. Anal. 32 (1995), 706-740. MR 96c:65162

[EJ3] K. Eriksson and C. Johnson, Adaptive finite element methods for parabolic problems IV: A nonlinear problem, SIAM J. Numer. Anal. 32 (1995), 1729-1749. MR 96i:65081

[EJT] K. Eriksson, C. Johnson and V. Thomée, Time discretization of parabolic problems by the Discontinuous Galerkin method, RAIRO, Anal. Numer. 19 (1985), 611-643. MR 87e: 65073

[FP] D.A. French and T.E. Peterson, A continuous space-time finite element method for the wave equation, Math. Comp. 65 (1996), 491-506. MR 96g:65098

[HW] E. Hairer and G. Wanner, Solving Ordinary Differential Equations II: Stiff and Differential-Algebraic Problems, Springer-Verlag, Berlin Heidelberg, Springer Series in Computational Mathematics v. 14, 1991. MR 92a:65016 
[HH] T.J.R. Hughes and G.M. Hulbert, Space-time finite element methods for elastodynamics: Formulations and error estimates, Comput. Methods Appl. Mech. Engrg. 66 (1988), 339-363. MR 89c:73060

[J] P. Jamet, Galerkin-type approximations which are discontinuous in time for parabolic equations in a variable domain., SIAM J. Numer. Anal. 15 (1978), 912-928. MR 80e:65102

[Jh] C. Johnson, Discontinuous Galerkin finite element methods for second order hyperbolic problems, Comput. Methods Appl. Mech. Engrg. 107 (1993), 117-129. MR 95c:65154

[KAD] O. Karakashian, G.D. Akrivis and V.A. Dougalis, On optimal order error estimates for the nonlinear Schrödinger equation, SIAM J. Numer. Anal. 30 (1993), 377-400. MR 94c:65119

[KM1] O. Karakashian and Ch. Makridakis, A space-time finite element mathod for the nonlinear Schrödinger equation: The discontinuous Galerkin method, Preprint no. 96-9, Dept. of Math., University of Crete, Heraklion, Greece (1996).

[KM2] O. Karakashian and Ch. Makridakis, A space-time finite element mathod for the nonlinear Schrödinger equation. II: The continuous Galerkin method, Preprint no. 96-11, Dept. of Math., University of Crete, Heraklion, Greece (1996).

[LR] P. Lesaint and P.A. Raviart, On a finite element method for solving the neutron transport equation, Mathematical Aspects of Finite Elements in Partial Differential Equations (C. deBoor, ed.), Academic Press, New York, 1974, pp. 89-123. MR 58:31918

[N] A.C. Newell, Solitons in Mathematics and Physics, vol. 48, SIAM, CBMS Applied Mathematics Series, 1985. MR 87h:35314

[RR] J.J. Rasmussen and K. Rypdal, Blow-up in nonlinear Schrödinger equations-I: A general review, Phys. Scripta 33 (1986), 481-497. MR 87m:35203

[SW] A.H. Schatz and L.B. Wahlbin, Interior maximum-norm estimates for finite element methods, Part II, Math. Comp. 64 (1995), 907-928. MR 95j:65143

[S] W.A. Strauss, The Nonlinear Schrödinger Equation, Contemporary developments in continuum mechanics and p.d.e.'s (G.M. de la Penha and L.A.J. Medeiros, eds.), North Holland, Amsterdam, pp. 452-465, 1978. MR 81i:35047

[T] V.I. Talanov, Self-focusing of wave beams in nonlinear media, JETP Lett. 2 (1965), $138-141$.

[Th] V. Thomée, Galerkin Finite Element Methods for Parabolic Problems, Lecture Notes in Mathematics, v. 1054, Springer -Verlag, Berlin, 1984. MR 86k:65006

[W] G.B. Whitham, Linear and nonlinear waves, Wiley, 1974. MR 58:3905

Department of Mathematics, University of Tennessee, Knoxville, Tennessee 37966

E-mail address: ohannes@math.utk.edu

Department of Mathematics, University of Crete, 71409 Heraklion, Crete, Greece

E-mail address: makr@sargos.math.uch.gr 